\title{
Mobile Game-Based Language Assessment
}

\author{
https://doi.org/10.3991/ijet.v15i03.11672 \\ Jan Delcker ${ }^{(\varpi)}$ \\ University of Mannheim, Mannheim, Germany \\ jan.delcker@bwl.uni-mannheim.de \\ Dirk Ifenthaler \\ University of Mannheim, Mannheim, Germany \\ Curtin University, Perth, Australia
}

\begin{abstract}
The goal of this article is to provide insight into the design, development and evaluation of a game-based language assessment tool for tablets. The overall objective was to create a mobile application for practitioners in kindergarten, enabling these practitioners to gather relevant language data from children aged three to five. An automated analysis of the data provides information about the child's language skills. If a need for support in language development can be found in the data, the application offers further possibilities how stakeholders can support a children's learning process for the specific field of language. The main criteria for a successful development were the effective collection of relevant data, high usability for practitioners and appropriate usage of a game-based mobile application for young children.
\end{abstract}

Keywords-Mobile tool, game-based assessment, language development, eyetracking, tablet

\section{Introduction}

Approximately one third of children enrolling in primary schools in Germany have an migration background [1]. The presence of a migration background is often linked to a language level which is less developed than the language level of same-aged children of German origin. When children grow up with one language at home (e.g., Turkish or Russian) and they get in contact with a second language in kindergarten, they are thought to be less efficient in the second language they are learning. Research shows that a correlation between language skills and the language spoken first cannot be made that easily. "The quality, frequency and type of language used in the home" [2] are main factors for the different development of language skills. If a child has a lot of experience and skills in his or her primary language, the child is more likely to develop higher skills in a second language [3]. Other important factors for the development of language skills are maternal characteristics [4] and the language used in the pre-schools and kindergartens the children are attending [5].

One of the main goals of the research project was the development of a spoken language level assessment tool for pre-elementary school children, aged three to five. The 
instrument should be able to collect reliable language data from the target group. From reliable data researchers can produce consistent, bias-free findings if the data is interpreted based on enough evidence and theoretical support. [6]. Regarding the developed instrument, statements about the language skills of an assessed child can be made. On the individual level, the tool can be used to identify educational needs of a child [7].

As a second goal, practitioners and parents are being provided with facilities, training methods and concepts to foster the child's further language development, based on the results of the assessment. In this way, participating families and institution can directly benefit from the use of the instrument [8]. The instrument is therefore designed to be more than a mere tool to gather data, hence, creating value for all stakeholders.

On a systemic level, the tool can be used to find specific differences regarding migration background, gender or age. Reaching this third goal enables stakeholders to develop systematic approaches to support groups of children with mutual development needs.

To reach the above mentioned goals, the instrument has to fulfill a number of specific requirements [9]. The collection of relevant data has to be parsimonious and the tool has to be easy to use for practitioners. Relevant assessment data in the context of spoken language assessment are statements made by the assessed user of a test instrument. To be able to assess those statements in the most efficient way, they should be recorded. The advantage of a recording over a live test is the fact that the data can be assessed by more than one time or by more than one person [10]. The quality of recorded speech data and language data in general is highly depended on the situation it is recorded in. Evidence based interpretations of language skills are relying on data that is representing real-life speech situations. The data has to be collected in situations which are as close to real-life situations as possible, with the least artificial distortions or disturbance possible. The avoidance of interferences to the speech situation is of special importance to the target group. Because the data is collected from young children, the natural speech situation is especially susceptible to derangement.

The ease of use or usability of the tool is important to increase both the amount of data gathered and the quality of the data. If the tool is easy to use, practitioners will use it more often and therefor gather more data. Additionally, a tool that is easy to use allows practitioners on concentrating on other important aspects, for example creating a stimulating text surrounding. A proper test setup results in a higher quality of gathered data.

Another important factor to reach the intended goals is the creation of an instrument that is engaging and interesting for children. A test tool that is fun to use increases the chances to create a natural speaking situation and support the gathering of reliable and valid data. The creation of a child-friendly, fun instrument helps to gain attention for the instrument and might reduce the need to advertise the instrument to stakeholders such as daycare centers, kindergartens and pre-schools. As a result, the amount of data that is available for analysis will increase. 


\section{Design}

The assessment of language skills, especially the ones of bilingual children, is a complex task [11]. One of the main issues is "the lack of (culturally) appropriate instruments" [12] that allow for the effective collection of reliable data. Given the goals, requirements and the target group presented in the preceding paragraph, the design of the instrument is based on reflections regarding a technology and game-based approach. A combination of those two aspects covers the main challenges of the research project, which made a team of professionals from the fields of linguistics, technology and education necessary for conducting the project [13].

Modern mobile technology like smartphones or tablets can be used as low priced recording tools that offer the recording quality necessary to create the language data for evaluation [14]. Besides the high functionality as recording devices, an internet connection makes it easy to collect the data, helping to prepare it for further analysis.

Additionally, an instrument that uses mobile technology is independent of a laboratory setting to gather data. This fact helps to work towards the goal of a test situation placed in a natural speech environment.

From a research-economic and research-administration perspective, designing a digital instrument rather than a paper-based requires a higher investment at the start of the project, for example the acquisition of equipment such as smartphones and tablets or financing the coding of a digital instrument. In the long-run, those expenses can be compensated, because the distribution of a digital instrument is very cost-efficient. In addition, changing the instrument in the future does not require the printing and distribution of new questionnaires or guidelines. While outdated versions of paper-based instruments have to be thrown away, digital instruments can be brought to the newest version by simply installing the updated version on any device in use.

While the technology based approach supports the goal to collect reliable spoken language data of high quality, the game-based approach to the instrument helps to create situations, in which this data is generated [15]. The target group of the research project, children in kindergarten and pre-school aged 3-5, spends a considerable amount of their time playing. Playing games and playful interactions with the environment are considered as of "vital importance for the healthy development of children" [16]. Because playing is a natural action for children and part of their daily activities, the statements made while playing can be considered as natural speech behavior or natural language data.

Playing games as a resource of natural language and using technology to record language can be combined in form of a video game. Being "interactive, simulated systems that are rule based, responsive, challenging, cumulative and inviting" [17], games offer a wide range of possibilities to create an assessment instrument for the language skills of children [18]. Initiating situations in which language is used to let the player interact with the game (and vice versa) captures the interactive and responsive characteristics of a game. Because the creator of the game is creating a simulated version of a situation, the creator has full control of the situation, how a story is developing or which reactions are caused by a specific action of the player. By designing a challenging and inviting game, the concentration of a player can be focused on specific tasks as well as the skills 
needed to succeed. As an assessment tool, a game offers the opportunity to measure and analyze those skills.

The instrument designed and developed for this project comprises of two applications working in conjunction with each other, combining the aspects of a mobile, gamebased instrument. The first application, called Wuschel App, is running on an iPad. Its main function is the collection of speech data from a participating child by playing a game. The second application is running on an iPhone or an iPod. The application is used by a practitioner to navigate through the game. The application is called Practitioners App. Although the instrument is presented in the form of a game, its main purpose is the assessment of a child's language skills and not entertainment. In the next chapter, both applications and how they interact with each other is explained more specifically.

\subsection{Game concept and graphics}

The game is designed as an adventure game. It is set in a magical forest. The main characters are Rita the Witch, Wuschel the Dog, Willy the Gnome and a mean dragon, archenemy of Rita. Throughout the game it is the child's task to protect Rita from the Dragon and to help her get specific objects. Rita is characterized as clumsy and she tends to lose important wizard items, for example her wand or her broom. The dog Wuschel is presented as her closest friend. With the help of the child, Wuschel is able to find the lost objects for Rita. Willy the Gnome is pranking Rita and Willy in a playful way, hiding objects from them. The graphics and the style of the game can be described as comic-like (see Figure 1). The drawing style of the characters and the setting is very child-friendly. By using bright and colorful patterns, the atmosphere is cheerful and stimulating. The game is constructed as a series of scenes, comparable to the pages of a children's book. In each scene, the child is presented with a specific task. After the situation of a sequence gets resolved, the next sequence can be started.

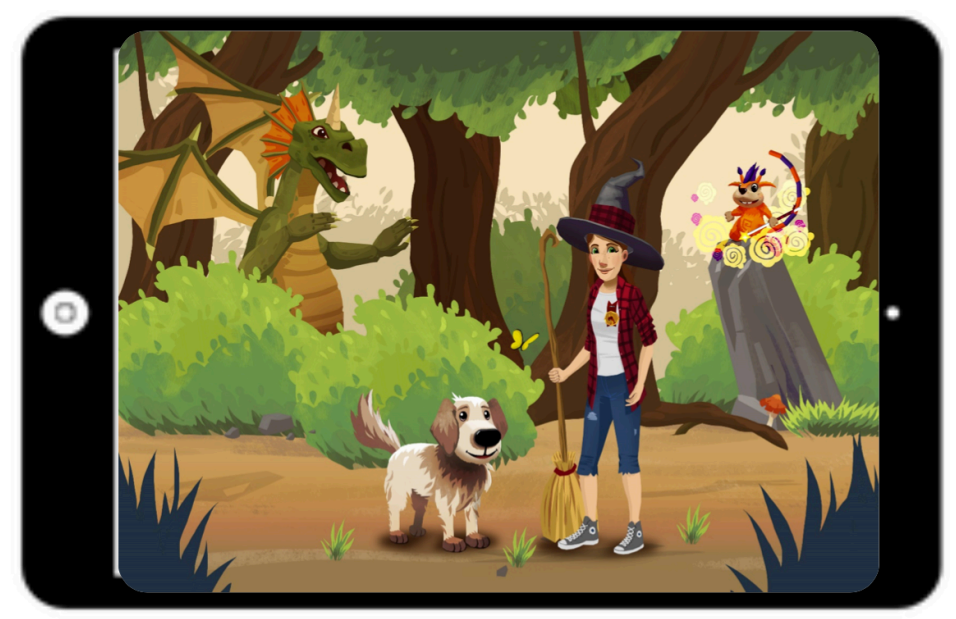

Fig. 1. Wuschel App 


\subsection{Tasks and controls}

In most cases, the objective of a specific scene is to find an object on the screen, for example the witch's wand. The tasks are presented by Wuschel by asking for the objects: "It looks like Rita lost her wand. Can you tell me where the wand is please?" The tasks are resolved when the player gives the right answer, for example: "The wand is on the kitchen table". If the right answer is given, Wuschel answers by saying in the sense of "Oh, I see it, it is over there!", walking to the objective and taking it. If the players answer is incorrect or if it is unclear, Wuschel restates the initial question: 'I'm not quite sure what you mean, can you repeat that please? Where can I find the wand?". One of the key aspects of the game is the fact that it is not actually speech-controlled. The scenes are pre-programmed and Wuschel will always find the objects at the end of the scene.

Hence, the children are not playing the game alone but are being supervised by a practitioner sitting in the background who is controlling the game with the Practitioner's App (see Figure 2). With the application a scene can be started, Wuschel's questions can be initialized and a scene can be finished. In this way, an adult keeps full control of the game, adapting the speed to the skills of the child playing the game. Because all the scenes are pre-scripted and Wuschel always finds the objectives or solutions, the flow of the story is not getting disrupted, which is preventing frustration and demotivation of the players.

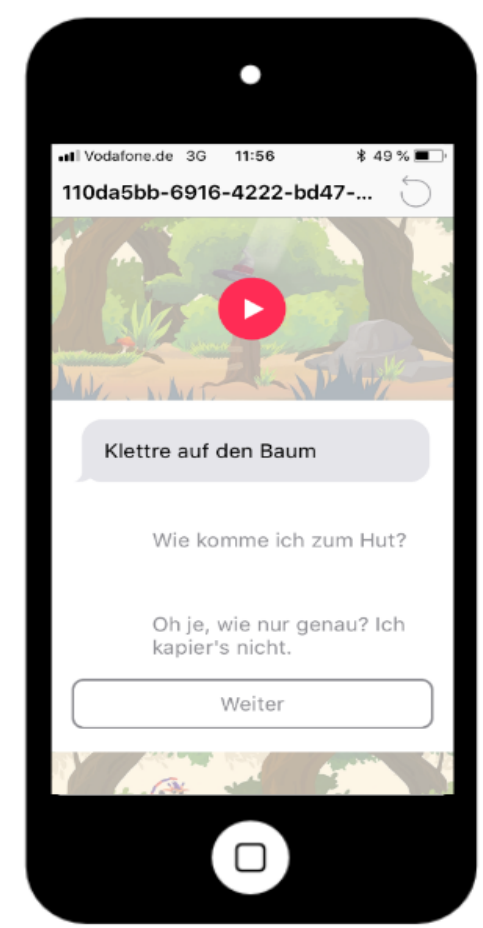

Fig. 2. Practitioner's App 
In the current state of the game, two types of tasks are implemented. The type is the localization of an object, for example the witch's wand as described in the preceded paragraph. The second type is the characterization of movement in form and direction. For the second type of task the player has to either describe a movement that was presented on the screen (e.g., "Willy fell from the tree!) or formulate a movement as a command for Wuschel (e.g., "You have to jump from stone to stone!"). The two types of tasks have been chosen because they create language data for prepositions. For linguist, those prepositions are of great significance, especially against the backdrop of bilingual children. Bilingual children might tend to mix prepositions from different languages.

\subsection{Data collection}

In preparation for the assessment, a legal guardian of the tested child has to complete an informed consent form, after being informed of the process of the assessment as well. Additionally, the legal guardians are asked to provide demographic data of their child (for example the age of the child and how long it has been speaking German). Each of the questionnaires and the informed consent forms contain a unique QR-Code and an 18-figure digital key. With the help of this key, consent form and personal data can be linked after the anonymization the data. This allows for the deletion of data if the legal guardians or the child decide to drop out of the research process at a later time (compliance with EU GDPR).

With the QR-Code, the personal data can be linked to the records of the Wuschel App. While the child is playing the game, the statements of the child are being recorded on the iPad. Each statement is saved as a separate file. After the completion of the game, the records are saved on a server, as soon as the iPad gets connected to a wireless network. The records are automatically linked to the provided biographic data of the assessed child via the digital key. In this way, the statements of a child can be matched with the biographical data without having to use the real name of the child. The safety and protection of the children's biographical and speech data is of upmost importance throughout the project.

\subsection{Data preprocessing and analysis}

After the recordings are saved on the server, instructed linguists transliterate the spoken language data into written form. The transcribed data can automatically be analyzed with linguist processing software that has been developed especially for this project. In the future, suggestions for treatment options or teaching programs will be provided by the application if practitioners want to receive them. In addition, automated transcription of speech to text is currently in development. 


\section{Usability of the Instrument}

Usability is defined as the "extent to which a system, product or service can be used by specified users to achieve specified goals with effectiveness, efficiency and satisfaction in a specified context of use" (ISO 9241-11:2018). It is a "quality attribute that assesses how easy user interfaces can be used" [19]. In the case of the developed instrument, the concept of usability has to be applied to a range of different aspects. (I) From a technological perspective, the instrument has to be working as intended. Technological functionality consists of the ability to easily install the software on the necessary hardware (iPad, iPod, iPhone) and to run it on those devices without crashes, independent of the version of the operating system. The usability is high, if the connection between the Wuschel App and the Practitioners App is working as intended and the data is successfully stored on the servers. (II) The operability of the Practitioners App is another part of the usability of the instrument. Practitioners have to be able to control the instrument from the application. The design of the application has to be clear and simple. All buttons have to be self-explaining. This way, practitioners can control the instrument independent of their ICT skills. The ease of use of the Practitioners App was of special interest for the design and development of the instrument. The instrument relies on practitioners being able to use the application to gather relevant data. (III) To ensure a high usability of the instrument, the Wuschel App has to be engaging and playful for the children. The development of a natural language situation is supported by the creation of an interesting story and appealing characters. Statements made in those situations are considered of more value by linguists, because they are closer to real-life language situations. Additionally, the instrument should be fun to use for all stakeholders.

The technical functionality of the instrument was tested before the other parts of the usability were reviewed. The instrument was run on the largest possible number of different devices and devices combinations to ensure flawless interaction between the Practitioners and the Wuschel App. The correct storing of language data on the servers was tested in the same way.

The usability of the Wuschel App was analyzed in great detail through multiple surveys. Initially, an eye-tracking survey was planned and conducted with children in a kindergarten. In a second step, students took part in an eye-tracking survey and a thinkaloud study.

\subsection{Increasing usability through game design}

During the design of the game, the priority was on the assessment focus. To ensure the gathering of relevant language data, the child has to have the impression of actually controlling the game with his or her voice. Most children, even at a young age are familiar with the touch sensitive displays of smartphones and tablets. One of the biggest challenges was to convince the children to use their voice and not their hands to control and navigate through the game. This challenge was even bigger, because the game is not controlled by the child at all but by a practitioner. Several design steps were implemented to ensure the child would have the impression it would control the game with 
his or her voice. In the first scenes of the game, in which the characters are introduced, the dog Wuschel shows the playing child how the interaction between the player and the dog is working. The witch Rita is giving instructions to the dog with her voice and Wuschel is following those instructions. Wuschel is underlining how he is reacting to Rita's spoken commands: "I am a good dog; I am always doing what I am told to do." As a second step, Wuschel is talking directly to child: "You should try it to. Tell me to dance and I will dance!". This is the first event in which the dog is directly responding to a command of the child. To further deepen the child's understanding of the control it is having over the dog, the player has to help the dog to get from one side of a small river to the other by jumping over from one stone to the other. At this event, Wuschel is clarifying that he can only hear, but not see the child and that the child has to give vocal commands. Wuschel is not reacting to gestures or interactions with the touch screen.

Apart from the desired behavior of the player to use voice commands to control the game, the assessed children were assumed to provide better data, if they would have fun playing the game. This requires the creation of challenging, yet solvable tasks. Willy the Gnome is used at different parts of the game to help the child understand what the solution to a problem is or to point out the location of an item.

\subsection{Usability testing of the wuschel app}

A game on a mobile device can only be interesting and engaging, if the player can see and understand everything that is happening on the screen. Hence, two eye-tracking surveys were conducted to ensure a clear visualization of the delivered content of the Wuschel App. Eye-tracking helps to understand at which part of a screen a person is looking at a specific moment. Although focusing on a part of the screen and understanding what is happening on the screen is not the same thing, research suggests that eye-tracking data can be used to make a connection between watching and comprehending $[20,21]$. While conducting an eye-tracking survey, a special camera, the socalled eye-tracker, is recording the eye movement of a user. With the help of specialized software, the eye-movements of the user are synchronized to the events on a screen. After the data collection, researchers are able to investigate on which parts of the screen the user was looking at. In the next step, statements about the visibility of objects and events on the screen can be made. The visibility of objects and events on the screen is vital to the usability of the instrument. A child can only make statements about objects and events in the game if it is able to see and recognize them. The eye-tracking studies were therefor used to identify critical events in the game which would have to be improved to increase the usability of the instrument.

The first eye-tracking study was conducted with five children in a kindergarten. The test environment was very close to the environment in which the instrument will be used in the future. A child is playing the game while a practitioner is controlling the game from the distance. During the study, a researcher took the role of a practitioner. A pre-school teacher was also present to make the child more comfortable. Soon after the eye-tracking study started, problems with the eye-tracking hardware became obvious. The tablets on which the Wuschel App was running had to be mounted on a special 
mobile device stand so the eye-tracker could make reliable recordings. This setup, which is normally used for mature participants of eye-tracking studies proved to be inapt for the young participants. The angle and the distance between the eye-tracker and the participant, the two most factors for reliable eye-tracking measurement, couldn't be held to the extent necessary. As a result, the collection of reliable eye-tracking data wasn't possible with children.

As an addition to the eye-tracking study at the kindergarten, a second eye-tracking study was performed with 20 adult students between 22 and 30 years. Although students are not the original target group of the instrument, eye-tracking could still be used to identify critical events in the Wuschel App. In addition, a think aloud study was conducted to gather information about the users impression of the Wuschel App. This information was used to identify weak points of the application which weren't of visual character.

The analysis of the eye-tracking data consists of an alignment between eye-tracking data and important objects. If the user is asked for information about a certain object on the screen, the focus of the user should be on that object shortly after the question is asked. For example, if the user is asked to find the witch's hat, the focal point should be on the area of the screen, where the hat is shown. If the focal point is not on the part of the screen where the hat is located, the user either can't see the hat or the hat cannot be identified as such.

During both the study with the children and the adult students all stationary objects and most moving objects were identified and denoted in the proper way. This suggests the assumption, that the visualization and the visibility of those objects was sufficient and would guarantee a high usability of the Wuschel App. To increase the usability further, the eye-tracking data of the objects which weren't identified correctly were observed in more detail. Two types of moving objects proved to cause difficulties for the user. The first type of objects which were hard to identify were those falling from the outside of the screen. The second type of objects were those which moved across from on one side of the screen and disappeared on the other. In both cases, the eyetracking data showed the user was looking at the objects when the first part of the movement (e.g., the entrance to the screen) already passed. It can be assumed that a longer screen time, caused by a slower movement, would increase the chance of the user focusing on the object. As a result, the movement speed of these objects was decreased in future versions of the application, further improving the usability of the whole instrument.

In the think-aloud study, the students described the overall design of the game as interesting and child friendly. All the instructions given by the dog were described as understandable, both in volume and presentation. The critical events identified with the eye-tracking study came also up in the think aloud study. Some students said, the movements were too fast and created a blur that made it hard to see and name the objects. The length of the story was criticized by some of the participants. One run-through takes around 30-40minutes, which was considered as to long for small children between three and five years.

Parallel to the eye-tracking study and the think-aloud study, the instrument was tested with 25 children in different pre-schools. The test was used to observe the overall 
interaction between the children and the game. The goal was to identify parts of the game in which children might be afraid or bored. Additionally, tasks which are to difficult should be found.

Overall, the children played the game with great interest. They showed excitement, moving close to the screen, paying close attention to the events, as well as expressing a range of emotions from laughter and joy to tension, but no fear or boredom. Although the run-through can be considered as long, no child decided to stop playing and everyone completed the game. The different tests conducted to survey the Wuschel App suggest that the usability of the application is very high. Weak points, such as the movement speed of objects, could be identified and improved.

\section{Discussion and Future Research Aspects}

At the current state of the research process, the declared research goals have only been reached partially. The design and development of a mobile, game-based instrument, that is capable of collecting reliable data can be considered as completed. The language data is reliably collected and of high quality. From a technological perspective, both applications are running without bugs and crashes. On the server-side, the language data is automatically stored and connected to the anonymized demographic data of the assessed children. Children's joy in playing the game and interacting with the characters on screen could be observed in the participating children.

Two main aspects will be in the focus of the future research process. In a first step, the automated transcription of the spoken language data has to be refined. Right now, training a transcription software to handle the data is one of the possible solutions for this task. Only after a reliable and efficient method of turning the language into an analyzable format is found, the linguist experts of the research group will be able to study and interpret the data using quantitative and qualitative methods. Furthermore, additional tasks will be added to the Wuschel App. On a systemic level, the influence of demographic variables on the development of spoken language skills can be explored. With those findings, programs can be developed to foster the development of second language children.

The second step of future research will be the provision of trainings, exercises and reading material once a certain need for support can be identified for a child. Using the existing system, feedback can anonymously be given to legal guardians and/or practitioners in the institutions.

Right now, one of the most critical issues is the distribution of the instrument to institutions for children aged three to five throughout Germany. Only if this distribution can be assured, the database used for future analysis will be filled with sufficient data. As of now, the applications are not publicly available. More important, most institutions do not possess the tools (i.e., iPad, iPhone, iPad) to use the applications. One idea to overcome those limitations is the creation of a mobile language lab. This lab consists of a suitcase which equipped with the necessary tools to conduct the assessment: an $\mathrm{iPad} / \mathrm{iPod}$ which carry the language assessment instrument and the paper-based demographic questionnaire as well as information material and the informed consent form. 
Institutions and practitioners will be able to order the suitcase and conduct the assessment after the shipping has arrived.

Once the limitations and future research goals have been tackled, the instrument offers an innovative way of reliably collecting spoken language data from children aged 3-5. Due to the implemented option of getting feedback about the language development of an assessed child, the instrument offers practical use for all stakeholders. Instead of being an instrument to collect data it can function as a tool to support children, legal guardians and practitioners to help understand the language development of children and furthermore foster their language learning progress.

\section{Acknowledgement}

The authors acknowledge the financial support by the Daimler and Benz Foundation.

\section{References}

[1] S. Bundesamt. (2018, 7.1.). Number of persons with migration background in Germany in 2017, by age (in 1,000). Available: https://www.statista.com/statistics/891872/personswith-migration-background-by-age-germany

[2] B. A. Collins, "Dual language development of Latino children: Effect of instructional program type and the home and school language environment," Early Childhood Research Quarterly, vol. 29, pp. 389-397, 2014/07/01/ 2014. https://doi.org/10.1016/j.ecresq.2014. $\underline{04.009}$

[3] R. L. Sparks, J. Patton, L. Ganschow, N. Humbach, and J. Javorsky, "Early first-language reading and spelling skills predict later second-language reading and spelling skills," Journal of Educational Psychology, vol. 100, pp. 162-174, 2008. https://doi.org/10.1037/0022$\underline{0663.100 .1 .162}$

[4] C. S. Hammer, E. Komaroff, B. L. Rodriguez, L. M. Lopez, S. E. Scarpino, and B. Goldstein, "Predicting Spanish-English Bilingual Children's Language Abilities," Journal of Speech, Language, and Hearing Research, vol. 55, pp. 1251-1264, 2012. https://doi.org/10.1044/1 $\underline{092-4388(2012 / 11-0016}$

[5] M. M. Páez, P. O. Tabors, and L. M. López, "Dual language and literacy development of Spanish-speaking preschool children," Journal of Applied Developmental Psychology, vol. 28, pp. 85-102, 2007/03/01/ 2007. https://doi.org/10.1016/j.appdev.2006.12.007

[6] R. Akbari, "Validity in Language Testing," in The Cambridge Guide to Second Language Assessment, C. Coombe, P. Davidson, B. O'Sullivan, and S. Stoynoff, Eds., ed: Cambridge University Press, 2012.

[7] A. K. Moeller, J. W. Creswell, and N. Saville, Second language assessment and mixed methods research. Cambridge: Cambridge University Press, 2016.

[8] A. Drigas, G. Kokkalia, and A. Economou, "Mobile Learning for Preschool Education," International Journal of Interactive Mobile Technologies (iJIM), vol. 10, p. 57, 2016. https://doi.org/10.3991/ijim.v10i4.6021

[9] L. F. Bachman, Statistical analyses for language assessment, 1. publ. ed. Cambridge [u.a.]: Cambridge Univ. Press, 2004.

[10] B. O'Sullivan, "Assessing Speaking," in The Cambridge Guide to Second Language Assessment, C. Coombe, P. Davidson, B. O'Sullivan, and S. Stoynoff, Eds., ed: Cambridge University Press, 2012. 
[11] A. Green, Exploring Language Assessment and Testing: Language in Action: Taylor \& Francis, 2013.

[12] T. Boerma and E. Blom, "Assessment of bilingual children: What if testing both languages is not possible?" Journal of Communication Disorders, vol. 66, pp. 65-76, 2017/03/01/2017. https://doi.org/10.1016/j.jcomdis.2017.04.001

[13] M. Shuib, A. Abdullah, S. N. Azizan, and T. Gunasegaran, "Designing an Intelligent Mobile Learning Tool for Grammar Learning (i-MoL)," International Journal of Interactive Mobile Technologies (iJIM), vol. 9, p. 41, 2015. https://doi.org/10.3991/ijim.v9i1.4238

[14] J. Delcker, A. Honal, and D. Ifenthaler, "Mobile Device Usage in Higher Education," in Digital Technologies: Sustainable Innovations for Improving Teaching and Learning, D. G. Sampson, D. Ifenthaler, J. M. Spector, and P. Isalas, Eds., ed, 2018, pp. 45-56. https:// doi.org/10.1007/978-3-319-73417-0_3

[15] C. S. Loh, Y. Sheng, and D. Ifenthaler, "Serious games analytics: Theoretical framework," in Serious games analytics. methodologies for performance measurement, assessment, and improvement, C. S. Loh, Y. Sheng, and D. Ifenthaler, Eds., ed New York, NY: Springer, 2015, pp. 3-29. https://doi.org/10.1007/978-3-319-05834-4 1

[16] D. G. Burac, "The Playful Behavior in Swimming and Its Interferences in 1-3 Years Child's Development," Procedia - Social and Behavioral Sciences, vol. 180, pp. 1229-1234, 2015/05/05/2015. https://doi.org/10.1016/j.sbspro.2015.02.252

[17] R. E. Mayer, Computer Games for Learning: An Evidence-Based Approach: MIT Press, 2014.

[18] X. Ge and D. Ifenthaler, "Designing engaging educational games and assessing engagement in game-based learning," in Handbook of research on serious games for educational applications R. Zheng and K. Garnder, Eds., ed Hershey, PA: IGI Global, 2017, pp. 255 - 272. https://doi.org/10.4018/978-1-5225-0513-6.ch012

[19] A. Dirin and M. Nieminen, "mLUX: Usability and User Experience Development Framework for M-Learning," International Journal of Interactive Mobile Technologies (iJIM), vol. 9, p. 37, 2015. https://doi.org/10.3991/ijim.v9i3.4446

[20] K. Conklin, Eye-tracking: a guide for applied linguistics research. Cambridge, New York, NY: Cambridge University Press, 2018.

[21] A. T. Duchowski, Eye tracking methodology: theory and practice, 2. ed. ed. London: Springer, 2007.

\section{$7 \quad$ Authors}

Jan Delcker is a German research assistant at the Chair of Learning, Design and Technology at the Area of Economic and Business Education of the University of Mannheim, L4, 1, in Mannheim, Germany. He graduated from the University of Applied Science Mannheim with a master's degree in social work, focusing on media education and quantitative social research. His current research interests are mobile learning and digitalistion in school development.

Dirk Ifenthaler is Professor and Chair of Learning, Design and Technology at University of Mannheim, Germany and UNESCO Deputy Chair of Data Science in Higher Education Learning and Teaching at Curtin University, Australia. His research focuses on the intersection of cognitive psychology, educational technology, data analytics, and organisational learning.

Article submitted 2019-09-13. Resubmitted 2019-10-09. Final acceptance 2019-10-10. Final version published as submitted by the authors. 